\title{
Medial temporal lobe function during emotional memory in early Alzheimer's disease, mild cognitive impairment and healthy ageing: an fMRI study
}

Mario A Parra ${ }^{1 *}$, Vivek Pattan², Dichelle Wong ${ }^{3}$, Anna Beaglehole ${ }^{4}$, Jane Lonie ${ }^{3}$, Hong I Wan ${ }^{5}$, Garry Honey ${ }^{5}$, Jeremy Hall ${ }^{3}$, Heather C Whalley ${ }^{3}$ and Stephen M Lawrie ${ }^{3}$

\begin{abstract}
Background: Relative to intentional memory encoding, which quickly declines in Mild Cognitive Impairment (MCI) and Alzheimer's disease (AD), incidental memory for emotional stimuli appears to deteriorate more slowly. We hypothesised that tests of incidental emotional memory may inform on different aspects of cognitive decline in $\mathrm{MCl}$ and $\mathrm{AD}$.

Methods: Patients with $\mathrm{MCl}, \mathrm{AD}$ and Healthy Controls $(\mathrm{HC})$ were asked to attend to emotional pictures (i.e., positive and neutral) sequentially presented during an fMRI session. Attention was monitored behaviourally. A surprise postscan recognition test was then administered.

Results: The groups remained attentive within the scanner. The post-scan recognition pattern was in the form of $(H C=M C l)>A D$, with only the former group showing a clear benefit from emotional pictures. fMRI analysis of incidental encoding demonstrated clusters of activation in para-hippocampal regions and in the hippocampus in $\mathrm{HC}$ and $\mathrm{MCl}$ patients but not in $\mathrm{AD}$ patients. The pattern of activation observed in $\mathrm{MCl}$ patients tended to be greater than that found in $\mathrm{HC}$.
\end{abstract}

Conclusions: The results suggest that incidental emotional memory might offer a suitable platform to investigate, using behavioural and fMRI measures, subtle changes in the process of developing AD. These changes seem to differ from those found using standard episodic memory tests. The underpinnings of such differences and the potential clinical use of this methodology are discussed in depth.

Keywords: Alzheimer's disease, Mild cognitive impairment, fMRI, Medial temporal lobe, Incidental memory, Emotional memory

\section{Background}

Memory and emotion are closely related to the functions of the Medial Temporal Lobe (MTL) and both are affected by Alzheimer's disease (AD). Episodic memory declines remarkably in patients with $\mathrm{AD}$ and in those with Mild Cognitive Impairment (MCI) [1-5]. The impact of $\mathrm{AD}$ on structures such as the hippocampus and

\footnotetext{
* Correspondence: mprodri1@staffmail.ed.ac.uk

'Scottish Dementia Clinical Research Network, Human Cognitive Neuroscience and Centre for Cognitive Ageing and Cognitive Epidemiology, University of Edinburgh, Edinburgh EH8 9JZ, UK

Full list of author information is available at the end of the article
}

the amygdala appears to be a responsible mechanism [6-11]. Functional Magnetic Resonance Imaging (fMRI) studies have suggested that AD-related changes seem to start very early and might express initially as a functional reorganization of MTL regions during episodic memory tasks [6].

There seems to be a relationship between the episodic memory impairment observed in patients with $\mathrm{MCI}$ and $\mathrm{AD}$ and their difficulties in processing emotional information. Emotional processing has been found to be sensitive to the effects of AD [12-17]. Patients with amnestic $\mathrm{MCI}$ and $\mathrm{AD}$ present with difficulties to

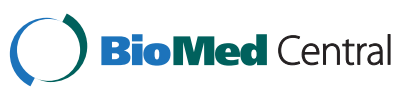


process emotional information in experimental and real life contexts [13,18-21]. Emotion is thought to support the formation of stable and durable representations of past experiences in long term memory [22]. Dere et al. [22] suggested that a deafferentiation of the hippocampus from emotional input, which might be required for episodic memory formation, could explain the development of memory and learning impairments throughout the course of $\mathrm{AD}$ [15]. The episodic memory impairment observed in $\mathrm{MCI}$ and early $\mathrm{AD}$ using explicit memory tasks frequently overlaps [23,24] albeit not all MCI patients will progress to AD. Therefore, the actual mechanisms underlying memory decline in these two conditions need further investigation. This knowledge can inform theoretical models which could help develop new clinical methods.

Functional measures of brain activity during incidental memory processing of emotional stimuli may offer a useful approach to address this issue. Incidental or implicit memory is commonly assessed by presenting participants with list of items (e.g., pictures, words, etc.) which they do not need to remember, and ask them later in a surprise test to recall or recognise the previously presented materials. The combination of emotional processing during implicit memory tests has already proved a valid approach to investigate whether episodic memory encoding can be enhanced in population with psychiatric disorders [25] (see also [26] for a recent meta-analysis on this methodology). Implicit memory seems to deteriorate much slower in the course of $\mathrm{AD}$ than explicit memory. This may offer opportunities to identify clear cut measures of AD progression. For example, MCI patients have shown preserved implicit learning functions in the presence of deteriorated explicit recognition [23]. Golby et al. [27] used fMRI to investigate explicit and implicit memory for natural scenes in AD. AD patients showed impaired explicit recognition memory but intact implicit memory for the scenes. The dissociation between impaired explicit and preserved implicit memory was found to be accounted for by encoding impairment in MTL and fusiform regions with intact implicit encoding in earlier-stage occipital cortex (see also [28-33] for evidence in support to this proposal), thus suggesting that $\mathrm{AD}$ impacts on the processing of emotion-related information differently depending on whether explicit/ intentional or implicit/incidental mechanisms are used to perform the task.

Based on the aforementioned literature, one might predict that functional assessment of MTL structures (e.g., parahippocampal gyrus - PHG, hippocampus, and amygdala) during incidental emotional memory may shed light into the differential impact of $\mathrm{MCI}$ and $\mathrm{AD}$ on these brain regions [34-36]. We are not aware of previous studies that combined these approaches to address this issue. The present study investigated this hypothesis in a sample of $\mathrm{AD}$ and MCI patients and in Healthy Controls. To this aim we chose a task previously reported by Whalley et al. [25] which investigates emotional memory for positive and neutral scenes using an incidental memory paradigm. We predicted a more evident decline in functional and behavioural measures of incidental memory for emotional stimuli in $\mathrm{AD}$ relatively to $\mathrm{MCI}$ patients and Healthy Controls. Based on previous structural and functional neuroimaging studies (e.g., $[15,17,25])$, we predicted differential activation of MTL structures between the groups.

\section{Methods}

\section{Participants}

In total 35 individuals were recruited. Three participants (one from each group) requested that the scan be stopped before completion of the emotional memory paradigm and hence provided no functional imaging data. Of the 32 individuals remaining, two subjects were subsequently excluded from analysis, one because of excessive movement during the scan ( $>3 \mathrm{~mm}$ per TR) and one due to excessive ventricular size. The research groups comprised 10 patients with $\mathrm{MCI}, 10$ patients with $\mathrm{AD}$ and 10 age-matched Healthy Controls (HC). The AD subjects were initially classified in accordance with the National Institute of Neurologic, Communicative Disorders and Stroke - AD and Related Disorders Association (NINCDS-ADRDA, [37]). Patients were recruited from tertiary referrals to the neuropsychological service or via referrals to the old age psychiatry service at the Royal Edinburgh Hospital. The MCI participants were recruited from tertiary referrals to the local neuropsychological assessment service for older adults and met criteria for amnestic MCI [38]. HC were recruited from the cohort study by Lonie et al. [39]. Six $\mathrm{HC}, 4 \mathrm{MCI}$ patients and $3 \mathrm{AD}$ patients were taking antihypertensive medications, $2 \mathrm{MCI}$ patients and $6 \mathrm{AD}$ patients were taking Acetylcholinesterase inhibitors.

Exclusion criteria for AD patients were other medical/ neurological conditions which could account for memory loss, untreated depressive illness, significant cerebrovascular disease on neuroimaging (i.e. exclusion of probable vascular aetiology) and significant motor or visual problems, or other factors which would preclude MRI scanning. Standard operational criteria for amnestic MCI were (1) memory complaint, corroborated by an informant; (2) abnormal memory function defined by memory performance of 1 standard deviation or more below age means on the delayed recall of one paragraph from the Logical Memory II subtest of the Wechsler Memory Scale-Revised [40]; (3) normal general cognitive function, as determined by a clinician's judgment based on a structured interview with the patient and an informant and a Mini-Mental State Examination (MMSE) 
score greater than or equal to 24 ; (4) no or minimal impairment in activities of daily living (ADLs), as determined by a clinical interview with the patient and informant; and (5) not sufficiently impaired, cognitively and functionally, to meet DSM-IV criteria for AD, as judged by an experienced AD clinician. We decided to use a less stringent threshold to assess objective cognitive decline in MCI (1 rather than 1.5 SD, but see [41] for recent guidelines) as we were interested in very early features of preclinical AD. Based on recent evidence [42], such boundaries may be difficult to delineate if higher cut-off scores are applied. Evidence from recent longitudinal studies supports the validity of this methodological approach [39]. The exclusion criteria were as described above. For the purposes of the current study to determine if any of the MCI patients should be classified as AD, or had returned to normal, a case summary was prepared describing clinical notes, current ACE and MMSE scores, and further background information as required, to determine current diagnostic status. Finally, the exclusion criteria for $\mathrm{HC}$ were a history of medical, psychiatric or neurological conditions that could affect cognitive functioning. $\mathrm{HC}$ group was matched to the MCI and AD groups in terms of age and gender. All the participants recruited into the study gave their informed consent. The study protocol (ref. 06/S1102/51) was reviewed and approved by the Lothian Local Research Ethics Committee 2, NHS. The demographic and psychometric characteristics of these groups are presented in Table 1.

\section{Scanning procedure}

Imaging was carried out at the Brain Imaging Research Centre (BIRC) for Scotland on a GE 1.5 T Signa scanner (GE Medical, Milwaukee, USA). The imaging protocol consisted of a localizer scan, followed by a T2-weighted fast spin-echo sequence, a structural T1 weighted sequence and a functional imaging paradigm: an emotional memory scan. The emotional memory task involved axial gradient-echo planar images $(\mathrm{EPI})(\mathrm{TR} / \mathrm{TE}=2500 /$ $40 \mathrm{~ms}$; matrix $=64 \times 64$; field of view $($ fov $)=24 \mathrm{~cm}$ ) acquired continually over two runs. Thirty contiguous interleaved 5-mm slices were aligned to the anterior and posterior commissure within each TR period. Each acquisition consisted of 99 volumes, of which the first four volumes were discarded. The T1 sequence yielded 128 contiguous $1.2 \mathrm{~mm}$ coronal slices (matrix $=192 \times 192$; fov $=24 \mathrm{~cm}$; flip angle $8^{\circ}$ ).

\section{Emotional memory paradigm}

The paradigm was that previously reported by Whalley et al. [25]. To construct the paradigm 72 images were selected from the International Affective Picture System [43], comprising 36 images depicting emotionally neutral scenes and 36 depicting emotionally positive scenes. Images were selected based on their valence and arousal. Mean normative control ratings for emotional valence (scored from 1 to 9 for most positive) and emotional arousal (scored from 1 minimum to 9 maximum) for the positive scenes were: 7.30 (std dev 0.52) and 5.72 (std dev 0.62) for set 1, and 7.12 (std dev 0.49) and 5.67 (std $\operatorname{dev} 0.62$ ) for set 2 . Images were divided in two sets for counterbalancing purposes (i.e., some participants were presented with set 1 within the scanner and set 2 was used as a distracter in the post-scan test and others received the opposite order). Emotional valence and emotional arousal ratings for the neutral scenes were 5.05 (std dev 0.47) and 3.07 (std dev 0.49) for set 1 and 5.14 (std dev 0.50) and 3.01 (std dev 0.59) for set 2. Similar valence and arousal values have been found to elicit significantly different responses across emotional categories $[44,45]$.

The scanning session was divided into blocks during which images of positive emotional scenes (emotion blocks), images of neutral emotional scenes (neutral

Table 1 Demographic and psychometric characteristics of the three groups of participants

\begin{tabular}{|c|c|c|c|c|c|c|c|c|}
\hline & \multirow{2}{*}{$\begin{array}{c}\text { Controls }(n=10) \\
M(S D)\end{array}$} & \multirow{2}{*}{$\begin{array}{c}M C I(n=10) \\
M(S D)\end{array}$} & \multirow{2}{*}{$\begin{array}{c}A D(n=10) \\
M(S D)\end{array}$} & \multicolumn{2}{|c|}{ ANOVA } & \multicolumn{3}{|c|}{ Bonferroni-corrected post-hoc tests ( $p$-values) } \\
\hline & & & & $F$ & $\overline{p \text {-values }}$ & $\mathrm{HC}$ vs. $\mathrm{MCl}$ & HC vs. AD & $\mathrm{MCl}$ vs. $\mathrm{AD}$ \\
\hline Age (years) & $74(8.89)$ & $76(9.03)$ & $78(7.56)$ & 0.57 & ns & ns & ns & ns \\
\hline Gender (M:F) & 4:6 & $3: 7$ & $5: 5$ & (Chi-sq & uare $=n s)$ & ns & ns & ns \\
\hline ACE-R score & $94.80(4.54)$ & $86.50(5.50)$ & $72.10(10.35)$ & 27.42 & $<0.001$ & 0.043 & 0.000 & 0.000 \\
\hline MMSE & $29.10(1.60)$ & $27.50(2.22)$ & $23.60(3.37)$ & 15.35 & $<0.001$ & 0.357 & 0.000 & 0.002 \\
\hline Attention and Orientation $(*)$ & $17.60(0.97)$ & $17.10(1.10)$ & $14.80(2.20)$ & 9.57 & 0.001 & 1.000 & 0.001 & 0.007 \\
\hline Memory $(*)$ & $23.90(2.23)$ & $17.90(3.96)$ & $11.30(4.37)$ & 29.96 & 0.000 & 0.003 & 0.000 & 0.001 \\
\hline Executive Functions $(*)$ & $12.70(1.49)$ & $11.00(1.33)$ & $8.20(2.66)$ & 13.98 & 0.000 & 0.175 & 0.000 & 0.009 \\
\hline Language $(*)$ & $25.60(0.70)$ & $24.90(1.60)$ & $23.10(2.51)$ & 5.33 & 0.011 & 1.000 & 0.011 & 0.092 \\
\hline Visuospatial functions $(*)$ & $15.40(0.70)$ & $15.60(0.52)$ & $14.10(1.66)$ & 5.65 & 0.009 & 1.000 & 0.037 & 0.014 \\
\hline NART & $118(7.70)$ & $116(6.88)$ & $108(10.60)$ & 4.35 & 0.028 & 1.000 & 0.024 & 0.148 \\
\hline
\end{tabular}

$\left(^{*}\right)$ Sub-scores of ACE-R. The minimum educational qualification achieved by the participants recruited into the study was high-school. 
blocks), and baseline fixation periods (baseline blocks) were presented. The baseline condition comprised the presentation of a fixation cross in the centre of the screen. Subjects completed two runs of the task each comprising 3 emotion blocks, 3 neutral blocks (25 s duration each) and 7 interleaved blocks of the baseline (12.5 s duration). For the emotion and neutral blocks six colour images were presented for $4 \mathrm{~s}$ each in random order and subjects were asked to simply press a button when they had seen the picture. This procedure was aimed at keeping arousal high within the scanner and to ensure that the participants were encoding the information presented. Was precisely this memory process (i.e., encoding) the one we investigated with this incidental memory paradigm ${ }^{\mathrm{a}}$. Subjects were given written and verbal instructions prior to scanning. In these instructions they were not asked to remember the presented images but just to press a button whenever a new image appeared on the screen. For the scanning component the delivery of stimuli was conducted using Presentation software.

Immediately after the scan a post-scan surprise recognition test was conducted on a laptop using E-Prime software. For the post-scan recognition memory test a further matched 72 images were selected as distracters following the counterbalancing procedure described above. Participants were then shown 144 slides (72 old items and 72 new distracters) and were asked to indicate whether the images have been seen within the scanner ('recognised') or whether they were new images ('not recognised'). They were asked respond by pressing two buttons previously assigned to each response. To further counterbalance old and new images and the order of presentation of the conditions, four parallel versions of the task were used, sets 1 and 2 with either neutral (e.g., set 1.1 or 2.1) or positive scenes (e.g., set 1.2 or 2.2) presented first. Pen and paper tests including the National Adult Reading Test (NART) of verbal IQ [46] and Addenbrooke's Cognitive Examination (ACE) [47] were also conducted after the scanning session.

\section{Behavioural analysis}

Within-scanner we recorded the number of responses to the stimuli. For the post-scan test, recognition memory was computed based on the percentage of corrected recognition (Hits - False alarms). Moreover, we implemented the Signal Detection Theory to calculate whether poor sensitivity $\left(\mathrm{A}^{\prime}\right)$ or a response bias $(\beta)$ could account for group differences in performance. Poor sensitivity would be informative of difficulties keeping signal (i.e., positive images) and noise (i.e., neutral images in memory) separate in memory. A negative response bias would indicate a tendency to choose the option "not seen before" in the post-scan recognition task, whereas a positive bias would reflect a tendency to choose the option "seen before". Statistical tests were conducted using SPSS for Windows (version 18.0, SPSS Inc., USA). Analysis of Variance (ANOVA) was used to test for differences between groups. When the dependent variable was not normally distributed, KruskalWallis non-parametric statistics was used. Bivariate Pearson Correlation analysis with behavioural and neuroimaging measures was also performed. This was aimed at identifying possible patterns of correlation between performance in the post-scan memory test and brain activation during incidental encoding in the region that proved most sensitive in group comparisons (i.e., MTL).

\section{Image processing for emotional memory paradigm}

EPI and T1 images were reconstructed offline into NIFTI format (Mayo Foundation, Rochester, MN, USA) using DICOM convert functions available in SPM5 (Statistical Parametric Mapping: The Wellcome Department of Cognitive Neurology and collaborators, Institute of Neurology, London) running in Matlab (The MathWorks, Natick, MA, USA). To assess data quality reconstructed images were examined using 'Art Repair' software (Centre for Interdisciplinary Brain Sciences Research, Stanford University, USA, http://cibsr.stanford.edu/tools/humanbrain-project/artrepair-software.html). For preprocessing, images were corrected for differences in image acquisition time between slices (slice timing) and then realigned to the mean functional image using a two-pass procedure to correct for movement artefact throughout the period of image acquisition. Normalization was performed to the EPI template available within SPM5 using standard preprocessing routines.

\section{fMRI within-groups analyses}

This was conducted using standard procedures using the general linear model approach as implemented in SPM5. At the individual participant level the data was modelled with the two conditions (emotion and neutral) each modelled by a boxcar function convolved with a synthetic haemodynamic response function. Estimates of head movement from the realignment stage of preprocessing were included as additional regressors in the model. Before fitting the model, the participants data was filtered in the time domain using high pass filter (128 s cut-off) and serial correlations were accounted for by using the autoregressive $(\mathrm{AR}(1))$ model. All preprocessing and analysis was conducted using default settings unless otherwise stated. Contrast images for each participant were then constructed for (i) all scenes versus baseline, (ii) emotional scenes versus baseline, (iii) neutral scenes versus baseline and (iv) emotional scenes versus neutral scenes. 


\section{fMRI between-groups analyses}

Contrast images were then entered into a second level random effects analysis to examine areas of activation within each of the three groups (one sample $t$ test), and differences in activation between the groups (ANOVA). Between group statistical maps were thresholded at a level of $\mathrm{p}=0.001$ uncorrected, and regions were considered significant at the cluster corrected threshold of $\mathrm{p}<$ 0.05 ( $\mathrm{K} e=10$ voxels). The latter is corrected for the chance of finding a cluster of this extent within the search volume and is therefore not reported in terms of FWE or FD. Based on our region of primary interest a small volume correction was used for MTL regions (amygdala, hippocampus and parahippocampal gyrus) based on the WFU PickAtlas [48,49].

\section{Results}

\section{Demographics and psychometric variables}

There were no significant differences between the groups in terms of age or gender, although there was a significant difference in NART IQ, and as expected, a significant difference in ACE and MMSE scores whereby AD patients scored more poorly than MCI and $\mathrm{HC}$ on these tests (Table 1). When the different subscores of ACE entered ANOVA, MCI patients showed a primarily amnestic deficit which persisted after controlling for IQ. AD patients however, showed significant deficits in all the subscores of ACE- $\mathrm{R}$ relative to both $\mathrm{HC}$ and MCI. For all the between group analyses presented below, IQ and gender were entered as covariates.

\section{Behavioural measures}

A two-way mixed ANOVA with Group as the betweensubjects factor ( $\mathrm{HC}$ vs. MCI vs. AD) and Block (Emotion vs. Neutral) as the within-subjects factor was first performed with the number of button press within the scanner. Mean data are shown in Figure 1a. There was no effect of Group $[F(2,27)=2.29$, n.s. $]$ or Block $[F(1,27)=$ 1.93, n.s.] nor was there a significant interaction between these factors $[F(2,27)=1.71$, n.s.]. A second ANOVA was carried out using the same model to analyse performance on the post-scan memory test. Mean data are shown in Figure $1 \mathrm{~b}$. There was a significant effect of Group $[\mathrm{F}(2,27)$ $=12.05, \mathrm{p}<0.001]$ whereby $\mathrm{AD}$ patients performed more poorly than both MCI (Mean Difference - MD = 31.66, $\mathrm{p}<0.05)$ and $\mathrm{HC}(\mathrm{MD}=48.61, \mathrm{p}<0.001)$. These last two groups did not significantly differ $(\mathrm{MD}=16.94, \mathrm{p}=$ 0.292). The effect of Block was also found to be significant $[\mathrm{F}(1,27)=8.96, \mathrm{p}=0.006]$ whereby the Emotion block resulted in better post-scan performance than the neutral block $(\mathrm{MD}=4.26, \mathrm{p}=0.009)$. The Group by Block interaction tended towards significance $[F(2,27)=$ 2.87, $\mathrm{p}=0.07]$.

Further post-hoc tests were performed to explore this tendency in more depth. Paired-sample t-tests carried out across blocks for each group separately confirmed that $\mathrm{HC}$ were the only group that showed an effect of emotion on performance whereby Emotional scenes resulted in better incidental memory than neutral scenes (HC: $\mathrm{MD}=8.33, \mathrm{t}=3.87, \mathrm{p}=0.004 ; \mathrm{MCI}: \mathrm{MD}=0.002, \mathrm{t}=$ 0.001, n.s.; $\mathrm{AD}: \mathrm{MD}=4.44, \mathrm{t}=1.58$, n.s.).
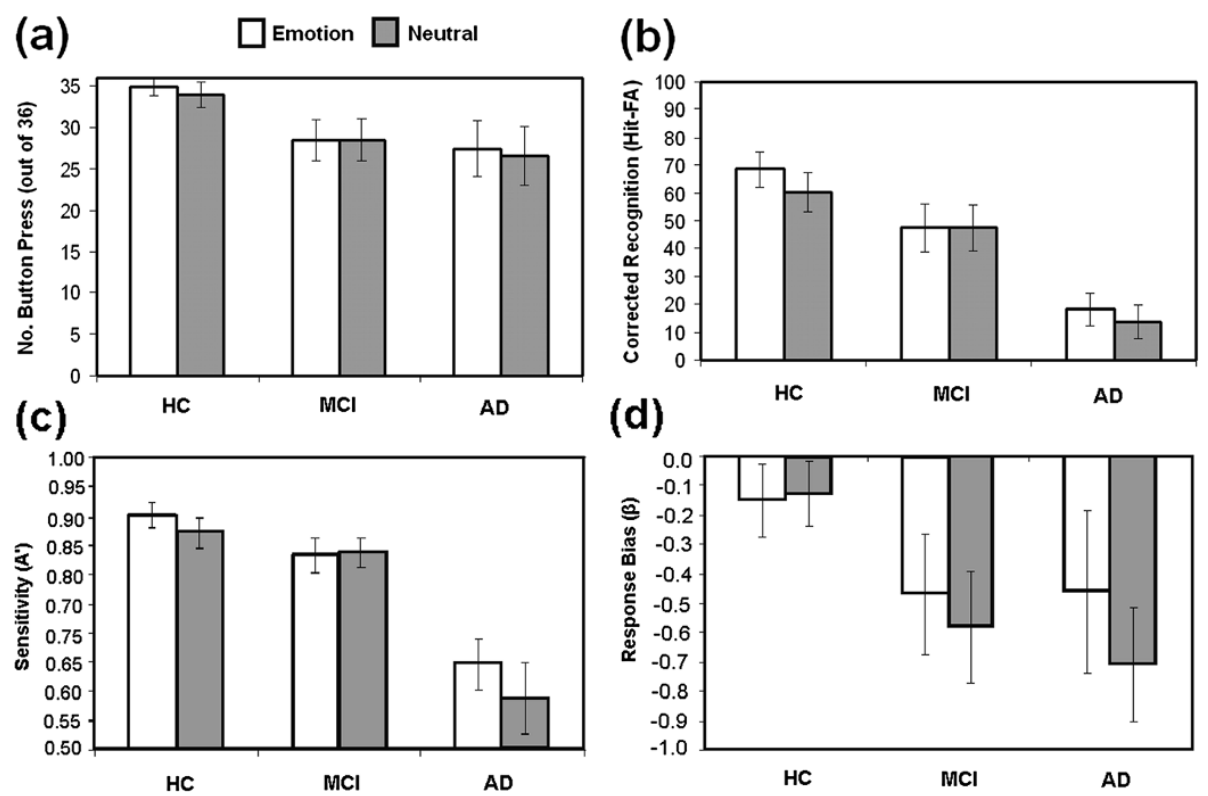

Figure 1 Behavioural data from the scanner and post-scanner emotional memory tests from the three groups of participants (error bars represent the standard errors of the mean). (a) Number of button presses while participants observed the images within the fMRI scanner. (b) Corrected recognition in the post-scan memory test. $(\mathbf{c} \& \mathbf{d})$ Variables of the Signal Detection Theory which inform on sensitivity $(\mathbf{c})$ and response bias $(\mathbf{d})$. 
The analysis using the Signal Detection Theory showed a significant effect of Group when A' was compared across Neutral images [Kruskal-Wallis test $=13.41$ $\mathrm{df}=2, \mathrm{p}=0.001$ ] and Positive images [Kruskal-Wallis test $=15.2 \mathrm{df}=2, \mathrm{p}=0.001] \quad$ (Figure 1c). AD patients were less able to keep separate signal from noise in memory relative to $\mathrm{HC}$ [Positive $=11.15, \mathrm{p}<0.001$; Negative $=13.5, \mathrm{p}=0.005$ ] and to MCI patients [Positive $=9.6, \mathrm{p}=0.044 ;$ Neutral $=11.1, \mathrm{p}=0.001]$. More negative response bias $(\beta)$ was observed for $\mathrm{AD}$ and MCI patients than for HC (Figure 1d). Neutral images tended to increase this negative bias in these groups. However, no significant effects were observed during the analysis of this variable.
fMRI measures: within-group analyses

Within group activations are presented in Figure 2 (for a fuller description see Additional file 1: Figure S1 and $\mathrm{S} 2$ ). For the within-group analyses with all scenes versus baseline, the $\mathrm{HC}$ and $\mathrm{MCI}$ group both demonstrated clusters of activation in MTL regions which were not observed in the $\mathrm{AD}$ group. Other regions of activation in $\mathrm{HC}$ and MCI group included medial frontal regions, insula, fusiform, cerebellum and occipital cortex. There was less activation overall in the $\mathrm{AD}$ group, but clusters were observed in fusiform, cerebellum and occipital regions. A similar pattern of findings was seen for the contrasts of emotional scenes versus baseline and neutral scenes versus baseline. However, the contrast of

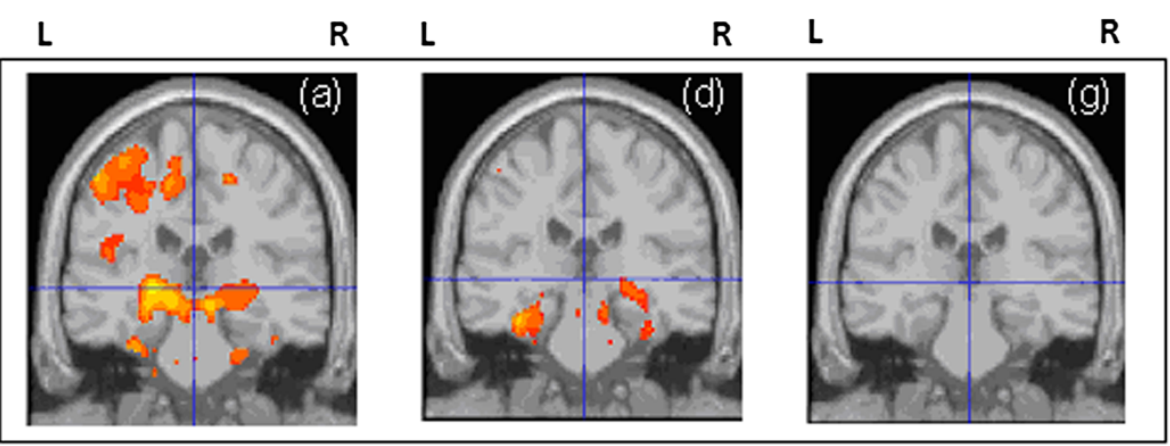

All scenes versus baseline

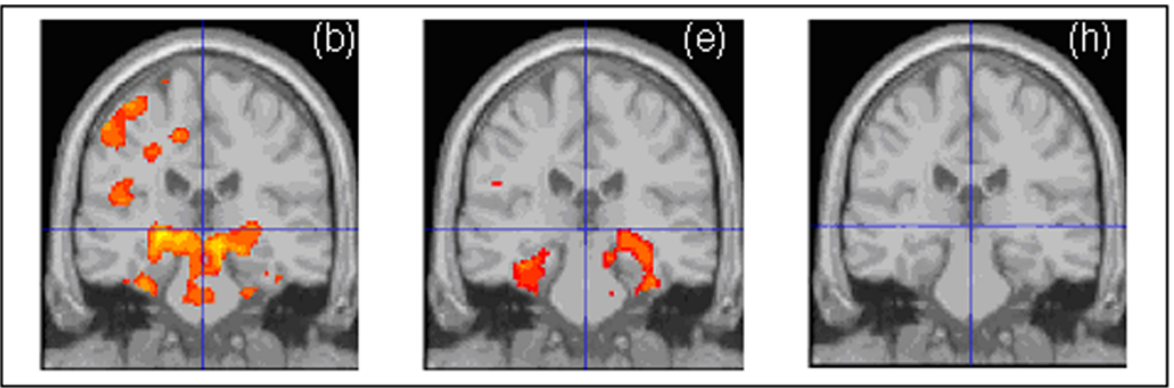

Neutral scenes versus baseline

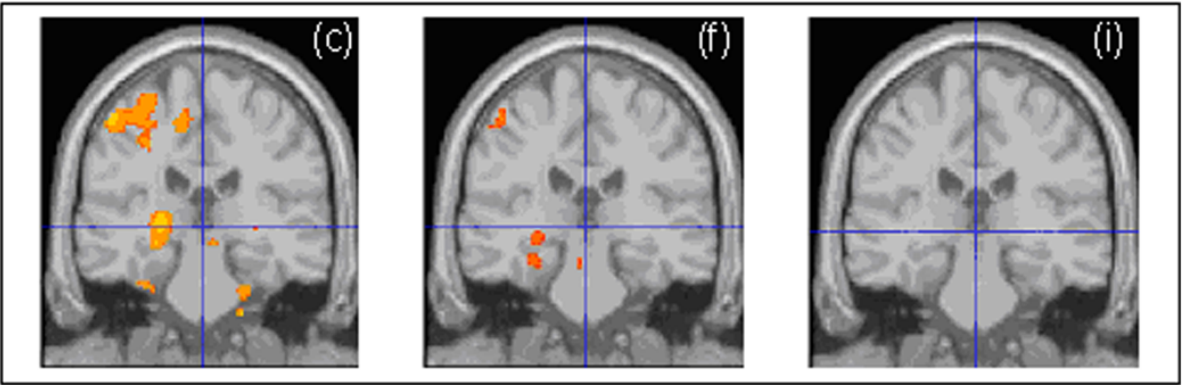

\section{Emotional scenes versus baseline}

Figure 2 Within group activation maps for $\mathrm{HC}(\mathbf{a}, \mathbf{b}, \mathbf{c})$, $\mathbf{M C l}$ patients $(\mathbf{d}, \mathbf{e}, \mathbf{f})$ and $\mathrm{AD}$ patients $(\mathbf{g}, \mathbf{h}, \mathbf{i})$. Images thresholded at $p=0.01$ uncorrected for illustration purposes only, all statistical comparisons performed at $p=0.001$ uncorrected threshold, as described in methods section. 
emotional scenes versus neutral scenes in controls did not yield significant differences.

\section{fMRI measures: between-group analyses}

Between groups results for all four contrasts examined are presented in Table 2. For the contrast of all scenes versus baseline, the $\mathrm{AD}$ group demonstrated decreased activation of the PHG/Hippocampal region versus both the controls $(\mathrm{p}=0.06)$ and MCI group $(\mathrm{p}=0.03)$ with the MTL small volume correction applied at threshold $\mathrm{p}<0.001$ uncorrected (see Figure 3 below). This pattern was also seen for the contrast of neutral scenes versus baseline, significant both against $\mathrm{HC}$ and $\mathrm{MCI}$ individuals. Again, a similar pattern of findings existed for the contrast of emotional scenes versus baseline but was below the chosen statistical threshold. There were no significant differences between the groups for the contrast of emotional scenes versus neutral. A graphical representation of the extracted data from the cluster of differences for the contrast of all scenes versus baseline is shown in Figure 4.

\section{Correlation with behavioural measures}

VOI values from the three groups were extracted from the contrasts where $\mathrm{MCI}>\mathrm{AD}$ in the MTL for each emotion versus baseline and neutral versus baseline separately. These contrasts were chosen because $\mathrm{HC}$ and MCI did not show significant differences in fMRI activation during the between-group analyses but both showed greater activation than the $\mathrm{AD}$ patients. These values along with corrected recognition data from the postscan test obtained from the three groups were entered in a bivariate Pearson correlation analysis. VOI values in the MTL during the encoding of Emotional pictures significantly correlated with performance in the post-scan test for Emotional $(\mathrm{r}=0.42, \mathrm{p}=0.02)$ and Neutral pictures $(\mathrm{r}=0.37, \mathrm{p}=0.04)$. When only $\mathrm{MCI}$ and $\mathrm{AD}$ patients were entered in the analysis, the correlation patterns described above persisted (Emotional pictures: $r=0.55, p=0.01$; Neutral pictures: $r=0.46, p=0.04$ ).

\section{Discussion}

The present study investigated whether functional and behavioural measures of incidental memory performance with emotional stimuli could help identify patterns of deterioration in MTL structures in MCI and AD patients. A profound decline in both functional and behavioural measures of episodic memory was found in the $\mathrm{AD}$ group relatively to $\mathrm{MCI}$ and $\mathrm{HC}$.

Within-scanner behavioural measures showed response rates over $90 \%$ for each group. No significant differences across groups were observed for these measures. Similar results were found by Whalley et al. [25] with a much younger group of participants. This suggests that despite the incidental nature of the task, the arousal within the scanner remained high throughout the assessment for the three groups of participants regardless of the content of the stimuli. The post-scan test however yielded different results. Memory performance was found to be worse in the AD group than in $\mathrm{MCI}$ and $\mathrm{HC}$ groups. Post-hoc tests confirmed that $\mathrm{HC}$ was the only group that showed a significant effect of the emotional content of the stimuli. Previous studies have suggested that emotional memory deteriorates in patients with MCI [19,21] and AD [12-17]. However, incidental memory for emotional stimuli has been less well investigated in $\mathrm{AD}$, particularly with the use of natural scenes. Previous studies reporting relatively preserved implicit functions in $\mathrm{AD}$ used eye movements recording [32] or tasks which did not have long intervals between encoding and test [31]. Our post-scan test was applied immediately after the scan. However, a few minutes lapsed in between these two phases. This may have led to decay of memory traces and attenuation of the facilitation elicited by the emotional content of the scenes in AD patients and to a lesser extent in MCI patients. However, HC could retain both more memory traces formed during the incidental task and the effects of the emotional pictures. The Socio-Emotional Selectivity Theory [50,51] posits that older adults' increasing awareness of time limitations motivates them to prioritize the seeking of emotional meaning over other goals. This can result in them

Table 2 Random effects analysis between groups

\begin{tabular}{lccccc}
\hline Group & P value & $\mathbf{K}_{\mathbf{E}}$ & $\mathbf{Z}$ & Co-ords & Region \\
\hline $\begin{array}{l}\text { All scenes versus baseline } \\
\text { HO>AD }\end{array}$ & 0.06 & 38 & 3.67 & $-28-26-16$ & Hippocampus/parahippocampal gyrus* \\
$\begin{array}{l}\text { MCl>AD } \\
\text { Neutral versus baseline }\end{array}$ & 0.03 & 73 & 4.25 & $-28-26-16$ & Hippocampus/parahippocampal gyrus* \\
HO>AD & & & & Hippocampus/parahippocampal gyrus* \\
MCl>AD & 0.02 & 119 & 4.65 & $-30-26-16$ & Hippocampus/parahippocampal gyrus*
\end{tabular}

Emotional versus baseline : $\mathbf{n} / \mathbf{s}$ (but clusters in similar locations to above at lower threshold)

Emotional versus neutral $: n / s$

IQ and gender entered as covariates into the analysis. Analysis thresholded at $p=0.001$ uncorrected. ${ }^{*}=$ small volume correction for MTL applied. $K_{E}=$ cluster size in voxels. 


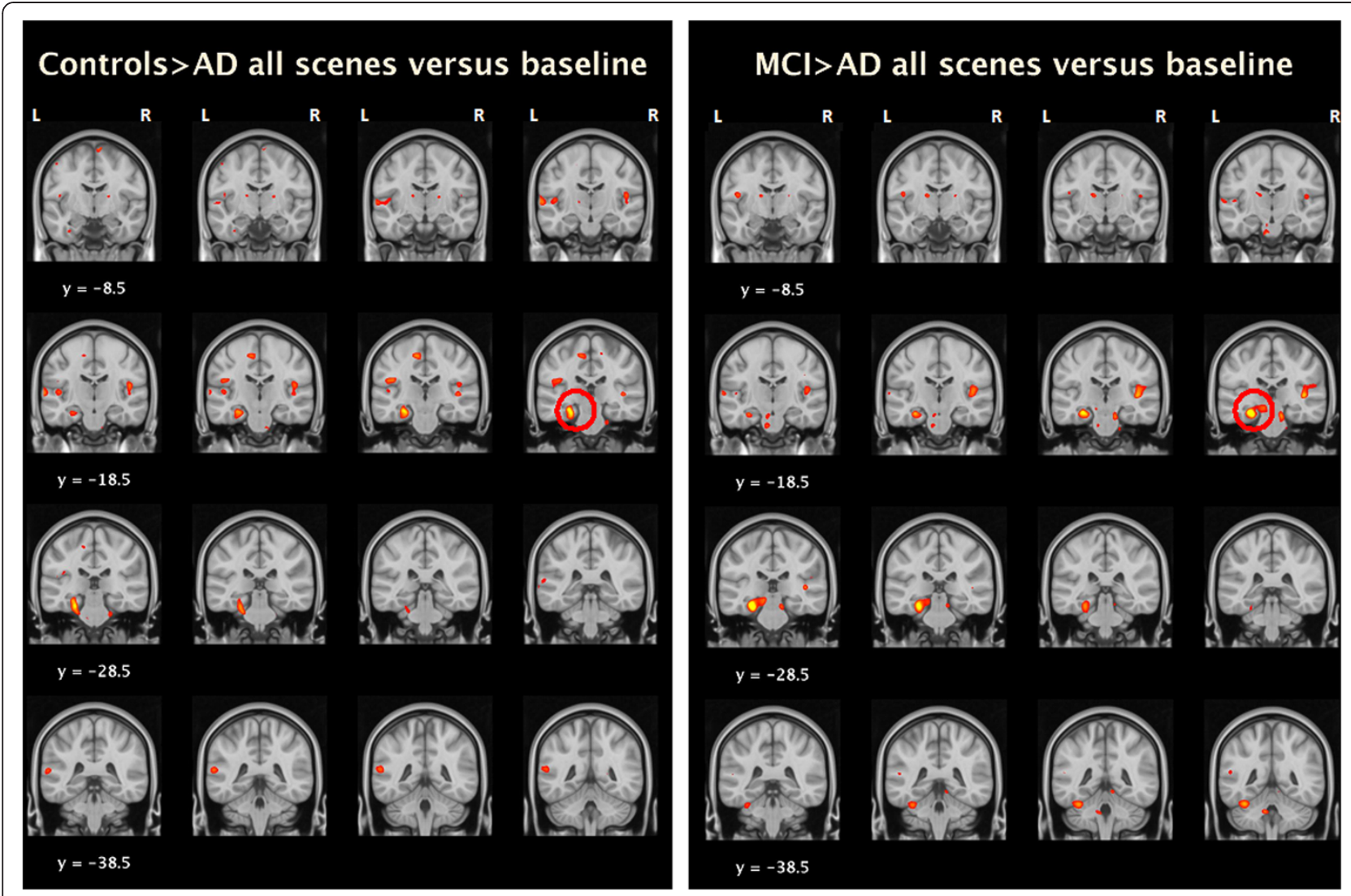

Figure 3 Between group activation differences (Colour scale red-yellow indicates T score, range 2.5-4).

prioritizing more positive and emotionally significant experiences a process that seems to be affected by AD.

Increased fMRI activity was found in $\mathrm{HC}$ and $\mathrm{MCI}$ groups in both MTL and medial frontal regions for the specific contrast of all scenes vs. fixation baseline but not in the AD group. Increased activation of the hippocampus has been previously reported in MCI patients

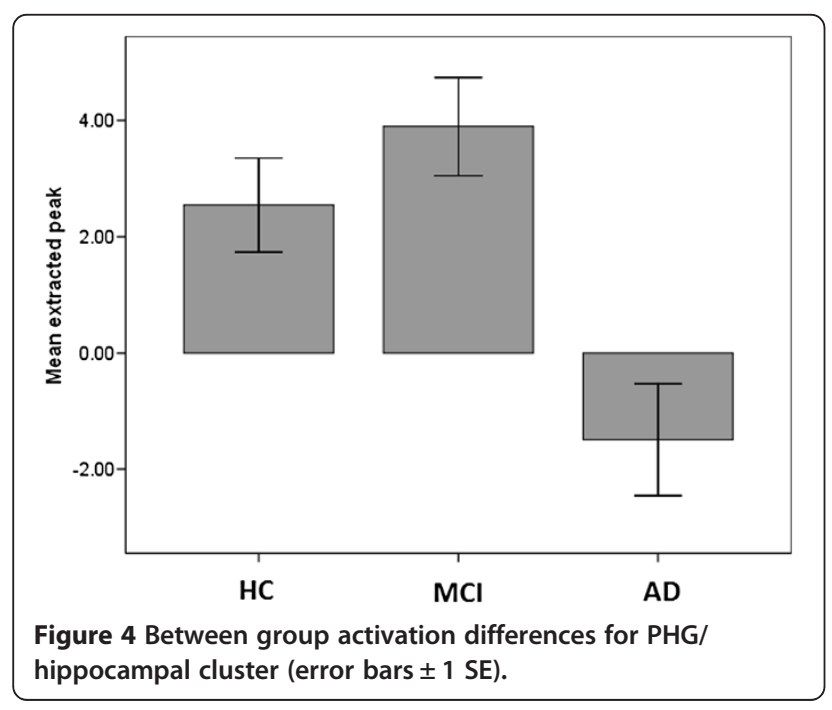

relative to cognitively intact individuals during associative memory tasks [52] and also in asymptomatic individuals who carry certain genotypes which lead to $\mathrm{AD}$ (e.g., E280A-PS1 [53] and APOE-e4 carriers [54]). Although fMRI differences between $\mathrm{MCI}$ and $\mathrm{HC}$ in our studies did not reach significance, the former group showed greater activation than the latter and was significantly different from AD patients. This seems to be in line with the suggestion of a compensatory phase early in the course of prodromal AD evidenced by increased MTL activation which is followed by a subsequent decrease as the disease progresses $[52,55,56]$. These memoryrelated changes in the hippocampal system might reflect a compensatory effort to overcome preclinical neural dysfunction caused by early pathological changes which do not reach threshold for behavioural expression. They suggest that hippocampal fMRI patterns during memory encoding may provide a preclinical biomarker for AD (see Quiroz et al. [53] for a similar view). Although our findings only hint to such mechanisms, future studies could investigate such a proposal using the present paradigm in a larger group of patients. Within and between group comparisons revealed that these activations were more significant for the contrasts of all scenes vs. baseline and emotional vs. baseline. A previous study which also used the emotional 
memory paradigm reported here suggested the usefulness of this methodology to investigate emotional-related decline in MTL structures in patients with schizophrenia and bipolar disorders [25]. However, they also acknowledged some limitations to this task. The authors claimed that positive scenes may be less uniformly arousing than negative emotional scenes. The block design used in the present study does not allow us to investigate whether the valence of some images selected from the IAPS was too subtle as to elicit differences in emotional processing at a functional level. The use of a block design may have resulted in the averaging of scenes of varying emotional intensity for a given subject within blocks. Furthermore, each block may include both remembered and forgotten pictures (see Whalley et al. [25] for a more thorough discussion on these issues) We thought it would be useful to consider these limitations as they could help us to better discuss our results within the current framework.

Reduced brain activation in MTL and frontal regions has been previously reported using explicit memory tasks in AD [27,57-60] and MCI [61,62]. However, the effects of emotional scenes on incidental learning in MCI and $\mathrm{AD}$ have not been thoroughly investigated using fMRI. As for the behavioural measures, the AD group showed the most impaired pattern of functional activation and together with the MCI group, they both showed no behavioural benefit derived from the emotional content of the stimuli. Potential accounts for these results were discussed above. An additional account for this functional impairment could be a poor encoding of emotional information. It might be possible that $\mathrm{AD}$ and MCI patients did not perceive the emotional content of the pictures as $\mathrm{HC}$ did. In fact, we screened this hypothesis in a subgroup of participants $(5 \mathrm{HC}, 7 \mathrm{MCI}$ and $3 \mathrm{AD}$ patients ${ }^{\mathrm{b}}$ ) which were asked to rate the images after the post-scan test as emotional or non-emotional. $\mathrm{AD}$ rated as emotional only $37 \%$ of the scenes presented within this category whereas they rated as neutral $84 \%$ of the neutral scenes. MCI patients rated as emotional $60 \%$ of the scenes and neutral $66.9 \%$. HC rated as emotional $75.5 \%$ and neutral $77.8 \%$. However, the perceptual impairment observed in this small group of patients did not hold during the functional assessment of the whole group. As we suggested above, the subtle difference across the emotional categories for some of the images chosen for the present study may account for the lack of a differential impact on functional activation. In fact, correlation analyses with behavioural and neuroimaging data showed that the relation between MTL activation and successful performance in the post-scan test across the two set of images was significant. An alternative explanation for the current findings could be that MTL regions are relevant to the overall episodic memory component of the current task but less so to its emotional component. MTL regions may be part of the network involved in the episodic memory processing of emotional stimuli but structures outside the MTL region may be more specifically involved in the emotional processing. In fact, the actual contribution of MTL regions (e.g., amygdale and hippocampus) to memory for emotional materials is not well understood. Different aspects of emotional stimuli (i.e., valance and arousal) seem to be processed by different regions of this network [63-65]. For example, the effect that the amygdale exerts on the hippocampus during emotional processing appears to be modulated by the arousal more than by the valence of the stimuli. Stimuli with low arousal do not activate the amygdale-hippocampus network [64] even if their valence can be accurately recognised [63]. Our emotional stimuli have high valence $(M=7.21, S D=$ $0.51)$ but low arousal $(M=5.7, S D=0.62)$. This may help explain the dissociation between behavioural and functional data in $\mathrm{HC}$ whereby effects of the emotional content was observed for the former but not for the latter data. AD appears to profoundly impact on the entire network which explains the lack of functional and behavioural changes across the two emotional dimensions as well as the dramatic drop in both measures relative to $\mathrm{HC}$. MCI patients may recruit areas adjacent to the functionally impaired regions as a compensatory mechanism [66]. These mechanisms would support the episodic component of the present task but not the emotional component. As for the AD group, the MCI patients failed to show emotional effects at both behavioural and functional levels even though their episodic memory was not poorer than that of $\mathrm{HC}$.

The evidence provided here warrants further investigation on this subject. The fact that both AD and MCI patients showed behavioural impairment in the emotional memory task but only the former group showed neuroimaging changes suggests that contrary to episodic memory, deterioration of emotional memory in these two conditions may have different neural correlates and may follow different trajectories (i.e., MCI may not always be preclinical AD, see Albert et al. [41]). These preliminary results in a small sample of MCI patients suggest that investigating the accuracy with which combined behavioural and neuroimaging data with the emotional memory paradigm could predict $\mathrm{MCI}$ to $\mathrm{AD}$ conversion would be worthwhile. Future studies may incorporate stimuli with negative valence or positive stimuli with higher arousal values. We predict that such manipulations would not undermine our primary findings, but they may shed new light on the actual involvement of the MTL network during emotional memory processing in $\mathrm{AD}$ and $\mathrm{MCI}$. Moreover, in the light of the limited number of studies available which have investigated emotional memory processing in $\mathrm{MCI}$ and $\mathrm{AD}$ 
using fMRI, we believe that the results presented here will stimulate future research on this subject. Neuroimaging biomarkers are becoming more reliable and widely incorporated in decision making algorithms for the clinical diagnosis of dementia [67]. When behavioural and functional (fMRI) measures of intentional memory are used, MCI and AD tend to show a great overlap [59] even though not all MCI patients convert to AD. This largely reflects the age-related decline of MTL structures. The ability to process arousing stimuli however seems to be preserved in old age [68]. Here we have shown that incidental emotional memory may help reduce the MCI/AD overlap. As we discussed above, this distinction might be disclosing age-unrelated cognitive decline which more reliably inform when MCI may be preclinical AD. Combining fMRI and the emotional memory paradigm reported here may offer a valuable tool for the preclinical assessment of risk for $\mathrm{AD}$ (see Reiman et al. [69] for recent evidence in familial AD).

Some limitations of the present study were discussed above (i.e., related to the stimuli used and the fMRI design). Another limitation is the sample size. Ten participants per group was enough to identify marginal effects in the two-way ANOVA with behavioural data $(\mathrm{p}=$ 0.074 , Power $=52 \%$ ). It is worth noting that conducting fMRI studies with patients with $A D$ is very challenging. This may be a factor explaining the limited number of studies available on the subject. Larger sample sizes will allow a better characterization of the effects of $\mathrm{MCI}$ and $\mathrm{AD}$ on emotional-related memory processing and MTL structures. Additionally, the use of an incidental memory paradigm in an fMRI environment with vulnerable older adults such as those with dementia may be other factors to be considered when designing fMRI studies. The effects of the fMRI environment on cognitive performance are now better understood [70]. Future studies might use scanner simulators to investigate these effects and to prepare the research participants prior to fMRI assessment.

\section{Conclusions}

Important clusters of activation were observed with the emotional memory paradigm in $\mathrm{HC}$ and MCI in MTL and medial frontal regions. These clusters were not observed in AD. AD and MCI patients showed behavioural impairment in the emotional memory task but only the former group showed neuroimaging changes. This suggests that contrary to episodic memory, deterioration of emotional memory in these two conditions may have different anatomical, functional and clinical implications. Whether a combined analysis of behavioural and neuroimaging data with the emotional memory paradigm could yield better predictive power to assess conversion to $\mathrm{AD}$ among $\mathrm{MCI}$ patients is subject which will require investigation.

\section{Endnotes}

${ }^{\text {a }}$ This task was the result of several pilot sessions. AD patients found it difficult or confusing to perform within the scanner versions of this memory task which required old/new responses or which assessed several memory processes (i.e., encoding, maintenance and retrieval).

${ }^{\mathrm{b}}$ After we had assessed a few participants, we noticed that patients, particularly of the MCI group, did not perceive all the emotional stimuli as such. Hence, we decided to test perception of our stimuli in the last participants recruited into the study.

\section{Additional file}

Additional file 1: Figure S1. All scenes versus baseline (Threshold $p<0.001)$. Figure S2. Contrast for the emotional versus neutral scenes (Threshold $p<0.001$ ).

\section{Competing interests}

$\mathrm{HCW}, \mathrm{AMM}, \mathrm{SML}$ and $\mathrm{JH}$ were in receipt of grant funding from the

Translational Medicine Research Collaboration, described above. HIW and GH were employees of Wyeth, now Pfizer. The other authors have no biomedical financial interests or potential conflicts of interest.

\section{Authors' contributions}

MAP: has made substantial contributions to acquisition, analysis and interpretation of data; has been involved in drafting the manuscript and revising it critically for important intellectual content; VP, DW, $A B, J L$ : have made substantial contributions to acquisition and interpretation of data and have been involved in revising the manuscript critically; HIW, JH: have made substantial contributions to conception and design and have been involved in revising the manuscript critically for important intellectual content; $\mathrm{HCW}$, SML: have made substantial contributions to conception and design, analysis and interpretation of data, have been involved in drafting the manuscript and revising it critically for important intellectual content; $\mathrm{GH}$ : has made substantial contributions to the interpretation of data, has been involved in revising the manuscript critically for important intellectual content. All authors read and approved the final manuscript.

\section{Acknowledgments}

This work was supported by an award (EU_036) from the Translational Medicine Research Collaboration, a consortium made up of the Universities of Aberdeen, Dundee, Edinburgh and Glasgow, the four associated NHS Health Boards (Grampian, Tayside, Lothian and Greater Glasgow \& Clyde), Scottish Enterprise, and Wyeth Pharmaceuticals. At the time of the work, JH was supported by a MRC Clinical Research Training Fellowship and AM was supported by the Health Foundation.

The study (E06419) was carried out at the SFC Brain Imaging Research Centre (www.sbirc.ed.ac.uk), and the Wellcome Trust Clinical Research Facility, University of Edinburgh, Western General Hospital, Edinburgh. The imaging was carried out on a 1.5 T Signa Horizon MR/I 1.5 T HDX operating under a research collaboration with GE Medical Systems (Milwaukee, USA), operating as IGE in the UK.

The Scottish Dementia Clinical Research Network supported MAP's work and the study in the identification of suitable patients. We also thank Klaus Ebmeier for his valuable comments on earlier drafts of our manuscript.

\section{Author details}

${ }^{1}$ Scottish Dementia Clinical Research Network, Human Cognitive Neuroscience and Centre for Cognitive Ageing and Cognitive Epidemiology, University of Edinburgh, Edinburgh EH8 9JZ, UK. ${ }^{2}$ Higher Specialty Trainee Old Age Psychiatry, Stobhill Hospital, Glasgow, UK. ${ }^{3}$ Division of Psychiatry, School of Molecular Medicine, Royal Edinburgh Hospital, Morningside, University of Edinburgh, Edinburgh EH10 5HF, UK. ${ }^{4}$ Old Age Psychiatry, St John's Hospital, West Lothian, UK. ${ }^{5}$ Translational Medicine, BioTherapeutics Clinical Programs, Pfizer, Inc, Cambridge, USA. 
Received: 31 May 2012 Accepted: 27 February 2013

Published: 6 March 2013

\section{References}

1. Dudas RB, Clague F, Thompson SA, Graham KS, Hodges JR: Episodic and semantic memory in mild cognitive impairment. Neuropsychologia 2005, 43(9):1266-1276

2. Fowler KS, Saling MM, Conway EL, Semple JM, Louis WJ: Paired associate performance in the early detection of DAT. J Int Neuropsychol Soc 2002, 8:58-71.

3. Greene JD, Baddeley AD, Hodges JR: Analysis of the episodic memory deficit in early Alzheimer's disease: evidence from the doors and people test. Neuropsychologia 1996, 34:537-551.

4. O'Connell H, Coen R, Kidd N, Warsi M, Chin AV, Lawlor BA: Early detection of Alzheimer's disease (AD) using the CANTAB paired Associates Learning Test. Int J Geriatr Psychiatry 2004, 19:1207-1208.

5. Swainson R, Hodges JR, Galton CJ, Semple J, Michael A, Dunn BD, Iddon JL, Robbins TW, Sahakian BJ: Early detection and differential diagnosis of Alzheimer's disease and depression with neuropsychological tasks. Dement Geriatr Cogn Disord 2001, 12:265-280.

6. Den Heijer T, Geerlings MI, Hoebeek FE, Hofman A, Koudstaal PJ, Breteler MM: Use of hippocampal and amygdalar volumes on magnetic resonance imaging to predict dementia in cognitively intact elderly people. Arch Gen Psychiatry 2006, 63(1):57-62.

7. Grady CL, Furey ML, Pietrini P, Horwitz B, Rapoport Sl: Altered brain functional connectivity and impaired short-term memory in Alzheimer's disease. Brain 2001, 124(Pt 4):739-756.

8. Heun R, Mazanek M, Atzor KR, Tintera J, Gawehn J, Burkart M, Gansicke M, Falkai P, Stoeter P: Amygdala-hippocampal atrophy and memory performance in dementia of Alzheimer type. Dement Geriatr Cogn Disord 1997, 8:329-336

9. Laakso MP, Soininen H, Partanen K, Helkala EL, Hartikainen P, Vainio P, Hallikainen M, Hanninen T, Riekkinen PJ Sr: Volumes of hippocampus, amygdala and frontal lobes in the MRI-based diagnosis of early Alzheimer's disease: correlation with memory functions. I Neural Transm Park Dis Dement Sect 1995, 9(1):73-86.

10. Markesbery WR, Schmitt FA, Kryscio RJ, Davis DG, Smith CD, Wekstein DR: Neuropathologic substrate of mild cognitive impairment. Arch Neurol 2006, 63:38-46.

11. Trivedi MA, Schmitz TW, Ries ML, Torgerson BM, Sager MA, Hermann BP, Asthana S, Johnson SC: Reduced hippocampal activation during episodic encoding in middle-aged individuals at genetic risk of Alzheimer's disease: a cross-sectional study. BMC Med 2006, 4:1

12. Cadieux NL, Greve KW: Emotion processing in Alzheimer's disease. J Int Neuropsychol Soc 1997, 3:411-419.

13. Kensinger EA, Brierley B, Medford N, Growdon JH, Corkin S: Effects of normal aging and Alzheimer's disease on emotional memory. Emotion 2002, 2(2):118-134

14. Mori E, Ikeda M, Hirono N, Kitagaki H, Imamura T, Shimomura T: Amygdalar volume and emotional memory in Alzheimer's disease. Am J Psyc 1999, 156(2):216-222.

15. Rosenbaum RS, Furey ML, Horwitz B, Grady CL: Altered connectivity among emotion-related brain regions during short-term memory in Alzheimer's disease. Neurobiol Aging 2010, 31:780-786

16. Satler C, Uribe C, Conde C, Da-Silva SL, Tomaz C: Emotion processing for arousal and neutral content in Alzheimer's disease. Int J Alzheimers Dis 2009 2009. doi:10.4061/2009/278615.

17. Schultz RR, De Castro CC, Bertolucci PH: Memory with emotional content, brain amygdala and Alzheimer's disease. Acta Neurol Scand 2009, 120:101-110.

18. Chaby L: Narme P: [Processing facial identity and emotional expression in normal aging and neurodegenerative diseases]. Psychol Neuropsychiatr Vieil 2009, 7:31-42.

19. Fujie S, Namiki C, Nishi H, Yamada M, Miyata J, Sakata D, Sawamoto N, Fukuyama $\mathrm{H}$, Hayashi T, Murai T: The role of the uncinate fasciculus in memory and emotional recognition in amnestic mild cognitive impairment. Dement Geriatr Cogn Disord 2008, 26:432-439.

20. Schroeter ML, Stein T, Maslowski N, Neumann J: Neural correlates of Alzheimer's disease and mild cognitive impairment: a systematic and quantitative meta-analysis involving 1351 patients. Neuroimage 2009, 47:1196-1206.
21. Teng E, Lu PH, Cummings JL: Deficits in facial emotion processing in mild cognitive impairment. Dement Geriatr Cogn Disord 2007, 23:271-279.

22. Dere E, Pause BM, Pietrowsky R: Emotion and episodic memory in neuropsychiatric disorders. Behav Brain Res 2010, 215:162-171.

23. Gronholm-Nyman P, Rinne JO, Laine M: Learning and forgetting new names and objects in MCI and AD. Neuropsychologia 2010, 48:1079-1088

24. Leube DT, Weis S, Freymann K, Erb M, Jessen F, Heun R, Grodd W, Kircher TT: Neural correlates of verbal episodic memory in patients with $\mathrm{MCl}$ and Alzheimer's disease-a VBM study. Int I Geriatr Psychiatry 2008, 23:1114-1118

25. Whalley HC, McKirdy J, Romaniuk L, Sussmann J, Johnstone EC, Wan HI, McIntosh AM, Lawrie SM, Hall J: Functional imaging of emotional memory in bipolar disorder and schizophrenia. Bipolar Disord 2009, 11:840-856.

26. Murty VP, Ritchey M, Adcock RA, LaBar KS: fMRI studies of successful emotional memory encoding: A quantitative meta-analysis. Neuropsychologia 2010, 48:3459-3469.

27. Golby A, Silverberg G, Race E, Gabrieli S, O'Shea J, Knierim K, Stebbins G, Gabrieli J: Memory encoding in Alzheimer's disease: an fMRI study of explicit and implicit memory. Brain 2005, 128(Pt 4):773-787.

28. Blessing $A$, Keil A, Linden DE, Heim S, Ray WJ: Acquisition of affective dispositions in dementia patients. Neuropsychologia 2006, 44:2366-2373.

29. Garcia-Rodriguez B, Fusari A, Rodriguez B, Hernandez JM, Ellgring H: Differential patterns of implicit emotional processing in Alzheimer's disease and healthy aging. J Alzheimers Dis 2009, 18:541-551.

30. Quoniam N, Ergis AM, Fossati P, Peretz I, Samson S, Sarazin M, Allilaire JF: Implicit and explicit emotional memory for melodies in Alzheimer's disease and depression. Ann N Y Acad Sci 2003, 999:381-384.

31. LaBar KS, Torpey DC, Cook CA, Johnson SR, Warren LH, Burke JR, WelshBohmer KA: Emotional enhancement of perceptual priming is preserved in aging and early-stage Alzheimer's disease. Neuropsychologia 2005, 43:1824-1837.

32. LaBar KS, Mesulam M, Gitelman DR, Weintraub S: Emotional curiosity: modulation of visuospatial attention by arousal is preserved in aging and early-stage Alzheimer's disease. Neuropsychologia 2000, 38(13):1734-1740.

33. Padovan C, Versace R, Thomas-Anterion C, Laurent B: Evidence for a selective deficit in automatic activation of positive information in patients with Alzheimer's disease in an affective priming paradigm. Neuropsychologia 2002, 40:335-339.

34. Appel J, Potter E, Shen Q, Pantol G, Greig MT, Loewenstein D, Duara R: A comparative analysis of structural brain MRI in the diagnosis of Alzheimer's disease. Behav Neurol 2009, 21:13-19.

35. Echavarri C, Aalten P, Uylings HB, Jacobs HI, Visser PJ, Gronenschild EH, Verhey $F R$, Burgmans S: Atrophy in the parahippocampal gyrus as an early biomarker of Alzheimer's disease. Brain Struct Funct 2011, 215:265-271.

36. Guo X, Wang Z, Li K, Li Z, Qi Z, Jin Z, Yao L, Chen K: Voxel-based assessment of gray and white matter volumes in Alzheimer's disease. Neurosci Lett 2010, 468:146-150.

37. McKhann G, Drachman D, Folstein M, Katzman R, Price D, Stadlan EM: Clinical diagnosis of Alzheimer's disease: report of the NINCDS-ADRDA Work Group under the auspices of Department of Health and Human Services Task Force on Alzheimer's Disease. Neurology 1984, 34:939-944.

38. Petersen RC, Smith GE, Waring SC, Ivnik RJ, Tangalos EG, Kokmen E: Mild cognitive impairment: clinical characterization and outcome. Arch Neurol 1999, 56:303-308.

39. Lonie JA, Parra-Rodriguez MA, Tierney KM, Herrmann LL, Donaghey C, O'Carroll RE, Ebmeier KP: Predicting outcome in mild cognitive impairment: 4-year follow-up study. Brit J Psychiat 2010, 197:135-140.

40. Wechsler D: Wechsler Adult Intelligence Scale-Third Edition (WAIS-III). San Antonio, TX: The Psychological Corporatio; 1997.

41. Albert MS, Dekosky ST, Dickson D, Dubois B, Feldman HH, Fox NC, Gamst A, Holtzman DM, Jagust WJ, Petersen RC, et al: The diagnosis of mild cognitive impairment due to Alzheimer's disease: recommendations from the National Institute on Aging-Alzheimer's Association workgroups on diagnostic guidelines for Alzheimer's disease. Alzheimers Dement 2011, 7:270-279

42. Morris JC: Revised Criteria for Mild Cognitive Impairment May Compromise the Diagnosis of Alzheimer Disease Dementia. Arch Neurol 2012, 69:700-708.

43. Lang PJ, Ohman A, Vaitl D: The International Affective Picture System (photographic slides). Gainesville: The Center for Research in Psychophysiology, University of Florida; 1988. 
44. Calkins AW, Deveney CM, Weitzman ML, Hearon BA, Siegle GJ, Otto MW: The effects of prior cognitive control task exposure on responses to emotional tasks in healthy participants. Behav Cogn Psychother 2011 39:205-220.

45. Lee TM, Lee TM, Raine A, Chan CC: Lying about the valence of affective pictures: an fMRI study. PLoS One 2010, 5:e12291.

46. Nelson HE, Willison JR: The Revised National Adult Reading Test-Test manual. Windsor, UK: NFER-Nelson; 1991.

47. Mioshi E, Dawson K, Mitchell J, Arnold R, Hodges JR: The Addenbrooke's Cognitive Examination Revised (ACE-R): a brief cognitive test battery for dementia screening. Int J Geriatr Psychiatry 2006, 21:1078-1085.

48. Maldjian JA, Laurienti PJ, Kraft RA, Burdette JH: An automated method for neuroanatomic and cytoarchitectonic atlas-based interrogation of fMRI data sets. Neuroimage 2003, 19:1233-1239.

49. Tzourio-Mazoyer N, Landeau B, Papathanassiou D, Crivello F, Etard O, Delcroix N, Mazoyer B, Joliot M: Automated anatomical labeling of activations in SPM using a macroscopic anatomical parcellation of the MNI MRI single-subject brain. Neuroimage 2002, 15:273-289.

50. Carstensen LL, Isaacowitz DM, Charles ST: Taking time seriously. A theory of socioemotional selectivity. Am Psychol 1999, 54:165-181.

51. Diamond LM, Aspinwall LG: Emotion Regulation Across the Life Span: An Integrative Perspective Emphasizing Self-Regulation, Positive Affect, and Dyadic Processes. Motivation and Emotion 2003, 27:125-156.

52. Dickerson BC, Salat DH, Greve DN, Chua EF, Rand-Giovannetti E, Rentz DM, Bertram L, Mullin K, Tanzi RE, Blacker D, et al: Increased hippocampal activation in mild cognitive impairment compared to normal aging and AD. Neurology 2005, 65(3):404-411.

53. Quiroz YT, Budson AE, Celone K, Ruiz A, Newmark R, Castrillón G, Lopera F, Stern CE: Hippocampal hyperactivation in presymptomatic familial Alzheimer's disease. Ann Neurol 2010, 68:865-875.

54. Filippini N, Maclntosh BJ, Hough MG, Goodwin GM, Frisoni GB, Smith SM, Matthews PM, Beckmann CF, Mackay CE: Distinct patterns of brain activity in young carriers of the APOE-epsilon4 allele. Proc Natl Acad Sci USA 2009, 106:7209-7214

55. Dickerson BC, Salat DH, Bates JF, Atiya M, Killiany RJ, Greve DN, Dale AM, Stern CE, Blacker D, Albert MS, et al: Medial temporal lobe function and structure in mild cognitive impairment.[see comment]. Ann Neurol 2004, 56(1):27-35

56. Woodard $J$, Seidenberg M, Nielson KA, Antuono P, Guidotti L, Durgerian S, Zhang Q, Lancaster M, Hantke N, Butts A, et al: Semantic memory activation in amnestic mild cognitive impairment. Brain 2009, 132:2068-2078.

57. Bassett SS, Yousem DM, Cristinzio C, Kusevic I, Yassa MA, Caffo BS, Zeger SL: Familial risk for Alzheimer's disease alters $\mathrm{fMRI}$ activation patterns. Brain 2006, 129(Pt 5):1229-1239.

58. Elgh E, Larsson A, Eriksson S, Nyberg L: Altered prefrontal brain activity in persons at risk for Alzheimer's disease: an fMRI study. Int Psychogeriatr 2003, 15(2):121-133.

59. Machulda MM, Ward HA, Borowski B, Gunter JL, Cha RH, O'Brien PC, Petersen RC, Boeve BF, Knopman D, Tang-Wai DF, et al: Comparison of memory $\mathrm{fMRI}$ response among normal, $\mathrm{MCl}$, and Alzheimer's patients. Neurology 2003, 61:500-506.

60. Sperling RA, Bates JF, Chua EF, Cocchiarella AJ, Rentz DM, Rosen BR, Schacter DL, Albert MS: fMRI studies of associative encoding in young and elderly controls and mild Alzheimer's disease. J Neurol Neurosurg Psychiatry 2003, 74:44-50.

61. Mandzia JL, McAndrews MP, Grady CL, Graham SJ, Black SE: Neural correlates of incidental memory in mild cognitive impairment: An fMRI study. Neurobiol Aging 2009, 30:717-730.

62. Sandstrom CK, Krishnan S, Slavin MJ, Tran T, Doraiswamy PM, Petrella JR: Hippocampal atrophy confounds template-based functional MR imaging measures of hippocampal activation in patients with mild cognitive impairment. Am J Neuroradio/ 2006, 27(8):1622-1627.

63. Berntson GG, Bechara A, Damasio H, Tranel D, Cacioppo JT: Amygdala contribution to selective dimensions of emotion. Soc Cogn Affect Neurosci 2007, 2:123-129.

64. Kensinger EA: Remembering emotional experiences: the contribution of valence and arousal. Rev Neurosci 2004, 15:241-251.

65. Kuchinke L, Jacobs AM, Grubich C, Vo ML, Conrad M, Herrmann M: Incidental effects of emotional valence in single word processing: an fMRI study. Neuroimage 2005, 28:1022-1032.
66. Sexton CE, Mackay CE, Lonie JA, Bastin ME, Terriere E, O'Carroll RE, Ebmeier KP: MRI correlates of episodic memory in Alzheimer's disease, mild cognitive impairment, and healthy aging. Psychiatry Res 2010, 184:57-62.

67. Sperling RA, Aisen PS, Beckett LA, Bennett DA, Craft S, Fagan AM, Iwatsubo $T$, Jack CR Jr, Kaye J, Montine TJ, et al: Toward defining the preclinical stages of Alzheimer's disease: recommendations from the National Institute on Aging-Alzheimer's Association workgroups on diagnostic guidelines for Alzheimer's disease. Alzheimers Dement 2011, 7:280-292.

68. Kensinger EA: Age differences in memory for arousing and nonarousing emotional words. J Gerontol B Psychol Sci Soc Sci 2008, 63:13-18.

69. Reiman EM, Quiroz YT, Fleisher AS, Chen K, Velez-Pardo C, Jimenez-Del-Rio M, Fagan AM, Shah AR, Alvarez S, Arbelaez A, et al: Brain imaging and fluid biomarker analysis in young adults at genetic risk for autosomal dominant Alzheimer's disease in the presenilin 1 E280A kindred: a casecontrol study. Lancet Neurol 2012, 11:1048-1056.

70. Gutchess AH, Park DC: fMRI environment can impair memory performance in young and elderly adults. Brain Res 2006, 1099:133-140.

doi:10.1186/1471-244X-13-76

Cite this article as: Parra et al:: Medial temporal lobe function during emotional memory in early Alzheimer's disease, mild cognitive impairment and healthy ageing: an fMRI study. BMC Psychiatry 2013 $13: 76$.

\section{Submit your next manuscript to BioMed Central and take full advantage of:}

- Convenient online submission

- Thorough peer review

- No space constraints or color figure charges

- Immediate publication on acceptance

- Inclusion in PubMed, CAS, Scopus and Google Scholar

- Research which is freely available for redistribution 